\title{
Developments of Corey-Chaykovsky in organic reactions and total synthesis of natural products
}

\author{
Majid M. Heravi ${ }^{1}$, Shima Asadi, Niousha Nazari, Boshra Malekzadh Lashkariani \\ ${ }^{1}$ Department of Chemistry, Faculty of Physics \&Chemistry Alzahra University, Vanak, Tehran, Iran.
}

\begin{abstract}
The reactions of sulfur ylides with carbonyl, olefin or imine compounds named as the Corey-Chaykovsky, provide oxiranes, cyclopropane or aziridine, respectively. In this report, we try to introduce this important name reaction and highlight a comprehensive overview on the applications of this useful reaction in synthetic organic chemistry and total synthesis of natural products. Dedicated to Professor Mohammad Reza Saidi, on the occasion of his honorable and decent retirement.
\end{abstract}

\section{Keywords}

Corey-Chaykovsky, Dimethylsulfonium methylide, Organic and total synthesis, Epoxidation, Dimethylsulfoxonium methylide 